\title{
Carnets
}

Revue électronique d'études françaises de l'APEF

Deuxième série - 2 | 2014

Frontières de la chronique

\section{Aux frontières de la Chronique : les Salons de Diderot (1769-1781)}

Jean-Christophe Rebejkow

\section{(2) OpenEdition}

\section{Journals}

\section{Édition électronique}

URL : http://journals.openedition.org/carnets/1307

DOI : 10.4000/carnets. 1307

ISSN : 1646-7698

Éditeur

APEF

\section{Référence électronique}

Jean-Christophe Rebejkow, "Aux frontières de la Chronique : les Salons de Diderot (1769-1781)», Carnets [En ligne], Deuxième série - 2 | 2014, mis en ligne le 30 novembre 2014, consulté le 19 avril 2019. URL : http://journals.openedition.org/carnets/1307 ; DOI : 10.4000/carnets. 1307

Ce document a été généré automatiquement le 19 avril 2019.

\section{(c) (i) (8)}

Carnets est mis à disposition selon les termes de la licence Creative Commons - Atribution - Pas d'utilisation commerciale 4.0 International. 


\title{
Aux frontières de la Chronique : les Salons de Diderot (1769-1781)
}

\author{
Jean-Christophe Rebejkow
}

1 La Chronique est à la croisée des genres : littérature, presse journalistique éphémère... Diderot, dans l'entrée *Chronique de l'Encyclopédie, reste très sceptique sur son utilité. On sait qu'il écrivit, sur la demande de son ami Grimm, des comptes-rendus d'expositions organisées par l'Académie royale de peinture et de sculpture, qui se tenaient tous les deux ans au Salon carré du Louvre : soit en tout neuf salons, de 1759 à 1781. Peut-on parler de «Chronique » à propos des Salons de Diderot?

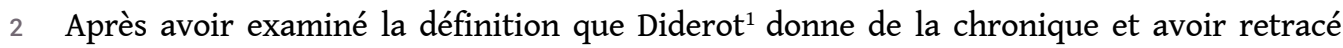
brièvement l'évolution de son sens, nous allons, dans un premier temps, nous demander en quoi les Salons qu'il écrivit, destinés à être diffusés dans la Correspondance Littéraire, revue manuscrite destinée aux têtes princières de l'Europe éclairée, relèvent de la Chronique, non pas tant au sens où l'entendait Diderot, mais au sens que ce mot a pris aujourd'hui.

3 Nous aimerions ensuite nous interroger sur l'insertion, au sein d'une "Chronique d'art ", d'allusions à des évènements contemporains ou quasi contemporains de l'écriture du Salon de 1769 qui semblent relever d'une autre «Chronique » (selon notre acception du XXI e siècle), celle de faits quotidiens, certes pas toujours " chronologique ", mais qui tranche avec l'écriture des salons précédents (1759-1765), dans lesquels, à côté de la description, Diderot s'intéressait essentiellement à l'essence de l'art, au «technique» du peintre. Cette Chronique semble nouvelle dans l'écriture des Salons. Que révèle précisément cette juxtaposition de «Chroniques », par rapport à l'évolution de la critique d'art de Diderot?

\section{Approches d'une définition de la Chronique : son lien avec le journalisme}

Le Système général de la connaissance humaine suivant le chancelier Bacon, dont les éditeurs de l'Encyclopédie sont largement redevables, fait de la Chronique une subdivision de l'Histoire: 
«Division de l'Histoire complète en Chroniques, Vies et Relations» (AT, XIII, p. 161). Diderot, auteur de l'article * Chronique de l'Encyclopédie (l'astérisque est la marque éditoriale de Diderot auteur), caractérise la Chronique comme événementielle, chronologique (« les faits abregés [...] [y] sont rangés selon l'ordre de leurs dates ») : elle est en ce sens synonyme d'annales (l'article Annales de l'Encyclopédie est de l'abbé Mallet).

La Chronique n'a pour Diderot que peu d'intérêt, à moins que le chroniqueur ne se double d'un historien, qui s'intéresse aux liens entre les "actions", plus qu'il ne s'attache à en relever le côté évènementiel et chronologique : "La Chronique, ne s'attachant qu'au gros des actions, ne sera pas fort instructive, à moins qu'elle ne parte d'une main habile qui sache, sans s'appesantir plus que le genre ne le demande, faire sentir ces fils imperceptibles, qui répondent d'un bout à des causes très-petites, \& de l'autre aux plus grands évenemens » (article * CHRONIQUE).

Aussi, après avoir évoqué les Chroniques des Égyptiens, des Juifs, les Chroniques des Saints, Diderot en vient à ce jugement lapidaire, et somme toute dépréciatif :

Le plus grand mérite de ces sortes d'ouvrages [les Chroniques des moines], dont les actions pieuses des saints ne font pas tellement l'objet, qu'on n'y trouve aussi les vies de plusieurs rois ou grands hommes, c'est d'avoir conservé les dates \& le fond des principaux évenemens. L'homme intelligent, qui sait rejetter le faux \& démêler le suspect, n'en tire que ce qui lui convient, \& peut-être n'en tire-t-il pas grandchose. (article ${ }^{*}$ CHRONIQUE).

On retrouve ici l'opposition du chroniqueur et de l'historien qui marquait le début de l'article. Un dictionnaire récent s'accorde avec Diderot sur ce point : « L'histoire implique, en théorie, une vision explicative, tandis que la Chronique est un simple enregistrement de faits, dans la succession des temps $»^{2}$.

C'est dans cette acception que le terme de Chronique est employé, dans les articles suivants de l'Encyclopédie : ABRÉGÉ, ARMES, BIDAUX [corps d' infanterie], IDEEN, Dactyle, JUIFS, Philosophie des («la Chronique des Samaritains », AT, XV, pp. 322, 323, 324). De même, la Correspondance Littéraire parle des "anciennes Chroniques d'Écosse " ${ }^{3}$. Dans son œuvre, notons par exemple que Diderot ne semble pas citer nommément les chroniqueurs du moyen âge.

Dans l'entrée *CHRONIQUE de l'Encyclopédie, et conformément à l'étymologie grecque (chronos : le temps), Diderot s'accorde avec l'article Chronique du Dictionnaire de l'Académie française: "CHRONIQUE. s.f. Histoire dressée suivant l'ordre des temps. Vieille Chronique. Anciennes Chroniques. Chroniques de Saint Denis. Il y a des mémoires de la vie de Louis XI qu'on appelle Chronique scandaleuse » (4e Edition, 1762 ; le sens de l'édition de 1694 est très proche). Le Dictionnaire critique de la langue française (1787-88) de Jean-François Féraud ne donne pas un autre sens à ce terme.

$10 \mathrm{Au} \mathrm{XVIII}$ siècle, le mot parait relativement peu employé. On le rencontre dans certains titres d'ouvrages, parmi lesquels : les Éphémérides du citoyen ou Chronique de l'esprit national que l'abbé Nicolas Baudeau (1730-1792), physiocrate, écrivit en collaboration avec le marquis de Mirabeau ; l'Histoire de Russie, tirée des Chroniques originales et des meilleures histoires de la nation, par P.-Ch. Levesque (1782-1783), mentionnée par Grimm dans la Correspondance littéraire d'octobre 1781, et louée en février 1782 (CL, XIII, pp. 35, 70 ss.). On peut citer également, dès la période révolutionnaire, la Chronique du mois, ou la Chronique de Paris fondée par l'archéologue Millin de Grandmaison (1759-1818), auxquelles collabora Condorcet. On rappellera enfin la Chronique scandaleuse (au XVIIIe s., celle d"Imbert, $c f$. Correspondance Littéraire, II, p. 151 ; XI, p. 80 ; XIII, p. 353, etc. $)^{4}$, Cette dernière acception 
du terme Chronique, non attestée dans les dictionnaires du temps, se retrouve dans la Correspondance Littéraire: il s'agit alors de la chronique d'évènements quotidiens ; c'est précisément en ce sens que Grimm, à qui Diderot s'adresse dans les salons, emploie ce mot, rapportant le terme de "Chronique " aux évènements qui jalonnent alors la vie quotidienne de Voltaire: "Autre chapitre de la Chronique de Ferney » (novembre 1769, CL, VIII, p. 365) Nous y reviendrons lors de notre analyse du Salon de 1769.

$11 \mathrm{Au} \mathrm{XIX}^{\mathrm{e}}$ siècle, la Chronique apparait comme genre littéraire, récit mettant en scène des personnages réels ou fictifs, les faits rapportés étant réels: citons par exemple les Chroniques italiennes et Le Rouge et le Noir, sous-titré Chronique du XIXe siècle de Stendhal (dans la Chronique de Charles IX de Mérimée, le terme semble pris dans son sens premier, originaire). Avec le dictionnaire de Littré (1863-1873; supplément en 1878), le terme de «Chronique » s'enrichit d'une nouvelle acception, encore usitée de nos jours : « 2. Fig. La Chronique, les Chroniques, ce qui se débite de petites nouvelles courantes ». En 1935, le Dictionnaire de l'Académie française fait le lien entre l'activité de chroniqueur et celle de journaliste : « Il se dit aussi de la Partie des journaux où l'on passe en revue les nouvelles $\mathrm{du}$ jour. Chronique politique. Chronique littéraire. Chronique musicale. Chronique mondaine. Chronique locale ». Le terme de "Chronique d'art » se rencontre chez Huysmans à propos de ses notes sur la peinture, en particulier pour le Salon de 1887. L'article *JOURNALISTE de Diderot dans l'Encyclopédie était déjà, à cet égard, révélateur :

Un journaliste est un «auteur qui s'occupe à publier des extraits et des jugements des ouvrages de littérature, de sciences et d'arts, à mesure qu'ils paraissent; d'où l'on voit qu'un homme de cette espèce ne ferait jamais rien si les autres se reposaient. Il ne serait pourtant pas sans mérite, s'il avait les talents nécessaires pour la tâche qu'il s'est imposée. Il aurait à cœur les progrès de l'esprit humain ; il aimerait la vérité, et rapporterait tout à ces deux objets » (AT, XV, p. 314).

12 Par cette attention portée à l'actualité, le journaliste est assimilé au critique ; et il n'est point étonnant de voir Diderot conclure ce même article *JOURNALISTE par ces mots: «Mais je m'aperçois qu'en portant ces observations plus loin, je ne ferais que répéter ce que nous avons dit à l'article CRITIQUE [de l'abbé Mallet]» (AT, XV, p. 316). On peut donc considérer le critique d'art en tant que journaliste, mais aussi en tant que chroniqueur, comme le souligne par ailleurs Le Dictionnaire du littéraire ${ }^{5}$.

\section{Chronique, journalisme et critique d'art : les Salons de Diderot}

13 La définition de la critique d'art reste très large et englobe, pour le $\mathrm{XIX}^{\mathrm{e}} \mathrm{s}$., différentes catégories qu'il faudrait préciser ${ }^{6}$.

14 Une autorité telle que Gita May n'hésite pas à qualifier les salons de Diderot de «Chroniques $»^{7}$. Diderot est aussi perçu comme chroniqueur d'art par d'autres éminents commentateurs ${ }^{8}$. Il s'agit alors de Chroniques dans une acception très large, et dans un sens peu restrictif.

15 En quoi les salons de Diderot s'apparentent-ils à la Chronique, aux Chroniques d'art ? Journaliste, Diderot l'est en rendant compte des salons pour ce périodique manuscrit qu'est la Correspondance Littéraire. Sans s'astreindre toutefois à un ordre chronologique strict, il tient compte de l'actualité, de la présentation des œuvres ${ }^{9}$; on peut retenir notamment, à cet égard, que Diderot est retourné en 1759 au salon pour voir la Médée de 
Carle Vanloo, «absente au début de l'exposition» (J. Chouillet, op. cit., p. 85). Il en va de même en 1761, pour L'Accordée de village, dont Greuze a retardé l'apparition, qui ne figure pas dans l'autographe ${ }^{10}$ du Salon de 1761, mais dont Diderot rendit compte dans la Correspondance Littéraire. Diderot s'intéressa de près aux débats de son temps. Dans le Salon de 1767, il s'insurgea contre le prix de sculpture décerné à Guillaume Moitte, prix qu'il attribua à la " cabale » de Vien, de Pigalle, et à la faiblesse de Le Moyne; Diderot aurait aimé qu'il revienne à un élève de Le Moyne, René Millot, qui l'obtint, en effet, en 1770 (AT, XI, p. 377). On pourrait ainsi multiplier les exemples. Ainsi, même si Diderot n'aime guère les chroniques, son activité de critique d'art qui tient compte de l'actualité des salons, autour d'un sujet précis, l'apparente à ce que nous considérons actuellement comme un chroniqueur.

Nous aimerions maintenant nous pencher sur le rôle de la « Chronique » dans les derniers Salons, en particulier dans le Salon de 1769, où une "Chronique » d'évènements qui émaillent le quotidien du philosophe s'ajoute à la «Chronique » d'art qu'il tient pour Grimm. Il convient d'abord de rappeler, ne fût-ce que brièvement, le rôle du Salon de 1767, qui marque, dans la réflexion de Diderot critique d'art, un véritable tournant.

\section{La mutation du projet esthétique de Diderot : le Salon de 1767}

Dans le Salon de 1767, le projet esthétique de Diderot change. Rappelons donc brièvement les principaux axes de cette mutation, étant donné qu'il ne saurait ici être question d'entrer dans les détails ${ }^{11}$ : nous allons voir que la réflexion sur le langage et la description constituent les facteurs principaux de l'évolution des idées esthétiques du philosophe dans ce Salon de 1767.

Le langage. La langue même se révèle insuffisante; les mots manquent au philosophe critique d'art pour répondre à la demande de Grimm: «J'abandonne une thèse, faute de mots qui rendissent bien mes raisons» (AT, XI, p. 179). Par ailleurs, les mots frappent maintenant Diderot non tant par leurs qualités plastiques (leur capacité à évoquer l'image) que par leurs qualités sonores, musicales:

J'en demande pardon à Marmontel, mais je n'ai jamais pu lire Lucain. [...] en dépit de la sublimité de l'idée, à ce sifflement aigu de syllabes Rheni mediis in fluctibus amnis ; à ce rauque croassement de grenouilles, quos inquinat, aequat, je bouche mes oreilles, et je jette le livre. (AT, XI, p. 327)

Malgré «la sublimité de l'idée " (c'est-à-dire aussi la beauté de l'image que le poète suggère), ce qui domine c'est ce « ce sifflement aigu de syllabes ». Un peu plus haut dans ce même salon, Diderot définissait déjà la poésie comme une " espèce de musique » (AT, XI, p. 270). La peinture et la poésie se révèlent ainsi dissemblables, même si Diderot s'interroge encore sur l'ut pictura poesis d'Horace (AT, XI, p. 72-73) ${ }^{12}$.

La description. On se souvient que, dès le Salon de 1761, Diderot en vient à penser qu'il peut rivaliser avec le peintre grâce à son style : « La couleur est dans un tableau ce que le style est dans un morceau de littérature ${ }^{13}$ ». Cette idée domine jusqu'en 1766. Dans le Salon de 1767, ses descriptions lui paraissent même monotones (AT, XI, p. 138, p. 221). Du reste, la description, même la plus simple, ne saurait suffire, elle doit être comparée à son «modèle ", le tableau : «Mais, encore une fois, quelle est la description d'un tableau de bataille qui puisse servir à un autre que celui qui l'a faite, les yeux devant le tableau [?]» 
(AT, XI, p. 186 ; à propos de Casanove). La même idée est reprise plus bas : « Voilà une description fort simple, une composition qui ne l'est pas moins et dont il est toutefois très difficile de se faire une juste idée, sans l'avoir vue» (AT, XI, p. 248). Autant dire que la description n'a plus de statut autonome, qu'elle ne parvient plus seule à évoquer l'image. Diderot est fatigué de décrire : "Je m'ennuie de faire et vous apparemment de lire des descriptions de tableaux. Par pitié pour vous et pour moi, écoutez un conte » (AT, XI, p. 204). Cette «lassitude » de la description (sensible dès les premières pages du salon), amène Diderot, dans le Salon de 1767, à envisager d'autres formes d'écriture : outre le conte des habitants du gros caillou (AT, XI, pp. 204 ss.), on peut noter la "Promenade Vernet » (AT, XI, p. 98 ss.), la Satire contre le luxe à la manière de Perse (AT, XI, p. 89 ss.) ${ }^{14}$, ou les commentaires sur la poésie...

Il n'est ainsi guère étonnant de voir Diderot, dans le Salon de 1769, écrire à Grimm : «Vous voyez, mon ami, que je vous fais grâce des descriptions, la partie qui m'amusait et qui prêtait à mon imagination » (AT, XI, p. 394). Que nous réservent donc les derniers salons, en particulier le Salon de 1769 ?

\section{La Chronique dans le Salon de 1769 et les Regrets sur ma vieille robe de chambre comme signe du désintérêt de Diderot pour la critique d'art}

Les Regrets sur ma vieille robe de chambre, diffusés dans la Correspondance Littéraire du 15 février 1769, sont antérieurs au Salon, qui eut lieu à l'automne de la même année. Diderot pensa cependant un moment intégrer ce texte à son salon, comme le prouve la mention sur le manuscrit autographe : "Fragment du sallon de 1769 ». Dans les Regrets sur ma vieille robe de chambre, Diderot se livre à une méditation sur l'esthétique, méditation qui prend comme prétexte le don que lui fit Madame Geoffrin d'une robe de chambre neuve, en remplacement de l'ancienne, usée : on peut voir ici l'intrusion d'une anecdote, qui relève de la chronique de la vie quotidienne. Cette anecdote informe le projet artistique de Diderot: la réflexion sur le rapport entre le luxe et les Beaux-Arts. Un des effets de ce luxe est de rompre l'harmonie du cabinet de Diderot: "Écoutez les ravages du luxe, les suites funestes d'un luxe conséquent. Ma vieille robe de chambre était une avec les autres guenilles qui m'environnaient»(AT, IV, p. 7). Mais, du fait de ce don, «Tout est désaccordé. Plus d'ensemble, plus d'unité. Plus de beauté » (AT, IV, p. 7) ${ }^{15}$. L'apparition de la nouvelle robe de chambre fait que le disparate règne maintenant en maitre dans la demeure du philosophe, et Diderot oppose ce disparate à l'unité du tableau de Vernet (AT, IV, p. 11 ss.). Les Regrets s'inscrivent ainsi d'une certaine façon dans la Chronique évènementielle, ici aux résonances autobiographiques (nous renouons ici avec le sens que donne Grimm à ce mot dans la Correspondance Littéraire à propos de Voltaire (CL, VIII, p. $365 ;$ cf. supra)).

Dans le Salon de 1769, Diderot reste toujours intéressé par l'actualité de la peinture, de la gravure ou de la sculpture, mais, à la différence des salons précédents, cette actualité s'inscrit plutôt dans la chronique évènementielle. Un exemple: la "mésaventure » survenue à Greuze, reçu certes à l'Académie, non comme peintre d'histoire, mais comme peintre de genre, pour ses productions passées, et non pour celle qu'il présentait comme son morceau de réception ${ }^{16}$ est relatée par Diderot dans une lettre à Sophie Volland du $1^{\mathrm{er}}$ octobre 1769 de la même année (AT, XIX, p. 326) et dans une lettre à Falconet du 7 
septembre 1769, dans laquelle il conclut ainsi : «Le fâcheux de tout cela, c'est qu'en effet le tableau ne vaut rien » (AT, XVIII, p. 317; son avis tranche avec celui qu'il exprimait précédemment, dans une lettre au même Falconet ${ }^{17}$ ). Par ailleurs, dans la conclusion du Salon de 1769, Diderot écrit à Grimm: "Vous ai-je dit que notre pauvre Académie de peinture a été sur le point de fermer son école ? Michel Van Loo n'est pas payé » (AT, XIX, p. 461) : la Correspondance Littéraire confirme le fait en avril 1771 (éd. Tourneux, IX, p. 288). Le Salon de 1769 présente d'autres traces d'une autre Chronique évènementielle, voire autobiographique, sans lien apparent avec la Chronique artistique; on peut citer, notamment :

- Le suicide de Desbrosses, dû à sa banqueroute (Salon de 1769, AT, XI, p. 420, p. 425), qui est également rapporté dans une lettre à Grimm du 19 novembre 1769 (LEW, VIII, p. 949). Rappelons que Desbrosses, est mis en scène dans Mystification ${ }^{18}$, récit qui relate une mystification réelle, restée inachevée à cause, précisément, de sa mort : Desbrosses devait faire comprendre à mademoiselle Dornet, un temps amante du prince Galitzine, qu'il fallait lui rendre un tableau; ce dernier pouvait se révéler compromettant pour le prince qui venait de se marier.

- La querelle sur l'édition de l'Encyclopédie, et les démêlés du philosophe avec Luneau de Boisgermain, qui considérait que les souscripteurs de l'Encyclopédie avaient été spoliés. Diderot prit le parti des libraires: «Après dîner je vais chez Briasson pour tâcher d'accommoder le procès de Luneau de Boisjermain avec les libraires » (AT, XI, 430). La lettre du 28.12.1768 (LEW, VIII, pp. 964-966) ${ }^{19}$ se fait l'écho de cette affaire.

- L'indisposition de sa fille Angélique (AT, XI, p. 440) est également évoquée dans une lettre du 12 novembre 1769 à Grimm (LEW, VIII, p. 951).

- Le remerciement à Grimm pour la « pacotille» de musique (AT, XI, 426).

Les évènements rapportés par Diderot sous forme d'anecdotes ne le sont pas forcément dans l'ordre chronologique. Cependant, la fonction de ces anecdotes, qui relèvent de la Chronique des évènements quotidiens, est différente dans les Regrets et dans le Salon de 1769. Alors que, dans les Regrets, l'anecdote (le don au philosophe d'une nouvelle robe de chambre par madame Geoffrin) était un prétexte à la vaticination de Diderot sur les méfaits du luxe et sur l'unité du tableau de Vernet, dans le Salon de 1769, l'anecdote semble plaquée, sans rapport avec la réflexion esthétique, et ne la nourrit pas (mis à part peutêtre l'allusion à un tableau, symbole de la compagnie des Indes (AT, XI, 448)). Les anecdotes semblent simplement former une Chronique qui se juxtapose à la Chronique picturale que tient Diderot pour Grimm. Ce mélange d'éléments disparates peut expliquer que Diderot, qui songea un moment à inclure les Regrets dans le Salon de 1769, y ait semblet-il finalement renoncé : dans la copie qu'il a fait faire de ses œuvres pour Catherine II, les deux textes sont séparés.

Il n'y a plus alors, dans le Salon de 1769, une description qui prétend se substituer aux tableaux, mais une simple juxtaposition sans lien apparent de remarques tenant lieu de chronique artistique, et d'éléments anecdotiques relevant d'une tout autre chronique: celle d'évènements parfois à caractère privé, contemporains de la rédaction de ce salon. Cette juxtaposition de deux chroniques signale exemplairement que Diderot a renoncé à rivaliser avec le peintre.

Le Salon de 1769 est ainsi marqué par l'intrusion d'éléments allogènes, étrangers au monde de la peinture, traces d'une autre Chronique, celle de la vie quotidienne. 
Les derniers salons (1771-1781) ne sont qu'un reflet affadi des premiers, de deux chroniques différentes. Le Salon de 1771 a paru déconcertant aux commentateurs.

Il frappe en effet par son disparate, qui repose sur la juxtaposition de deux "Chroniques d'art », de deux points de vue différents, voire opposés ${ }^{20}$. A une lettre sur les œuvres exposées font suite des notations brèves (mises entre parenthèses), censées être de Diderot lui-même, jugements lapidaires et le plus souvent dépréciatifs, qui reprennent en fait les jugements de Daudé de Jossan dans sa Lettre de M. Raphael le jeune [...] à un de ses amis, architecte à Rome (s.l., 1771). La lettre qui ouvre le Salon de 1771 semble prétendument adressée à l'éditeur de la Correspondance Littéraire, mais son ton appliqué (AT, XI, p. 465), qui tranche avec la liberté de ton des salons précédents ${ }^{21}$, a fait considérer ce salon par les critiques comme étant en partie l'œuvre d'un littérateur sollicité par Diderot : à moins qu'il s'agisse d'une nouvelle mystification du philosophe, qui fait suite à celle des notations entre parenthèses de ce même salon? Aucun des avis ne semble prévaloir dans le salon de 1771, et cette juxtaposition nous parait également une nouvelle preuve du fait que Diderot a renoncé à rivaliser avec le peintre. Le Salon de 1775 reconduit ce procédé sous forme d'un dialogue théâtral cette fois - qui semble caractéristique de la dernière manière de Diderot critique d'art: Daudé de Jossan y est d'ailleurs à nouveau sollicité. Dans le Salon de 1781, ce ne seront plus des observations d'un autre chroniqueur, mais des notes d'un soi-disant copiste (Meister? on sait que Meister avait pris la direction de la Correspondance Littéraire à la suite de Grimm ${ }^{22}$ : mais la démarche est comparable. Diderot s'en remet à l'opinion dans le Salon de 1781 : à propos de la Madeleine pénitente de Van Loo, il écrit par exemple: «Je crois ce tableau mal dessiné ; je m'en rapporte sur ce point à ceux qui en savent là-dessus plus que moi » (LEW, XIII, p. 94).

Dans les derniers salons, le disparate des chroniques, plutôt succession de monologues que dialogue réel, l'emporte donc finalement sur l'harmonie d'ensemble qui régnait jusqu'en 1765 dans les compte rendus écrits pour Grimm : confirmation du désintérêt progressif de Diderot pour la critique d'art.

$\mathrm{Au} \mathrm{XVIII}^{\mathrm{e}}$ siècle, la Chronique est considérée comme événementielle, simple récit d'évènements passés classés dans un ordre chronologique. Dans l'article * ${ }^{*}$ CHRoniQue de l' Encyclopédie, Diderot la dévalorise, et semble opposer le chroniqueur à l'historien, qui, lui, s'interroge avec profit sur les liens entre les évènements. Dans son œuvre, il emploie d'ailleurs fort peu le terme de "chronique», le réservant essentiellement à la relation d'évènements passés.

Les dictionnaires du temps, en particulier le Dictionnaire de l'Académie, partagent cette conception de la chronique comme «Histoire dressée suivant l'ordre des temps », et citent en exemple des Chroniques des temps anciens. Ce n'est qu'au XIX ${ }^{e}$ et au $\mathrm{Xx}^{\mathrm{e}}$ siècles que le terme de Chronique revêt d'autres significations.

On peut penser que Diderot, dans ses salons, s'apparente à un chroniqueur, comme en témoignent le côté chronologique, daté des salons, les allusions aux débats de l'époque et aux évènements qui émaillent le petit monde de la peinture (vente de tableaux, peintres corrompus par l'or...). Diderot est d'ailleurs considéré de nos jours par certains commentateurs comme un chroniqueur d'art. Dans cette perspective, il nous a paru intéressant, après avoir rappelé les raisons qui ont poussé Diderot dès 1767 à abandonner 
son projet de rivaliser avec le peintre par la description, de nous pencher sur ses derniers salons, et de voir le rôle qu'y joue la chronique. Le Salon de 1769, qui mêle Chronique artistique et "Chronique du quotidien », nous parait symptomatique de l'évolution des conceptions de Diderot. On se souvient que, dans les Regrets sur ma vieille robe de chambre, texte que Diderot songea un moment à incorporer au Salon de 1769, l'anecdote (le don par madame Geoffrin d'une nouvelle robe de chambre) alimente la critique d'art, la nourrit, en suggérant à Diderot des réflexions sur le rapport entre le luxe et les Beaux-Arts, entre l'unité du tableau de Vernet, qu'il oppose au disparate de son appartement ; mais le Salon de 1769, par la simple juxtaposition d'éléments relevant de chroniques de natures différentes, est un signe manifeste de l'abandon du projet de Diderot: rivaliser avec le peintre, et une manifestation du désintérêt de Diderot pour la critique d'art. Les anecdotes, qui relèvent d'une chronique du quotidien (la mort de Desbrosses, la maladie de sa fille Angélique, les démêlés avec Luneau de Boisgermain à propos de l'édition de l' Encyclopédie), n'informent plus la réflexion esthétique du philosophe. Les derniers salons confirment cette désaffection, par la juxtaposition de deux chroniques d'art, où tout dialogue semble exclu: le jugement de Diderot, le plus souvent lapidaire, ne prime d'ailleurs plus.

L'évolution de la chronique, dans les derniers salons, signale ainsi exemplairement l'abandon du projet esthétique de Diderot.

\section{BIBLIOGRAPHIE}

\section{Textes du XVIII ${ }^{\mathrm{e}}$ siècle}

Chronique de la Régence et du règne de Louis XV (1718-1705) ou Journal de Barbier (1875). Paris :

Charpentier.

DIDEROT (1875-1877) Euvres complètes, édition publiée par Jules Assézat et Maurice Tourneux.

Paris : Garnier frères, 20 vol.

DIDEROT (1969-1973). Cuuvres complètes, édition publiée sous la direction de Roger Lewinter. Paris :

Le Club français du livre, 15 vol.

DIDEROT (2003). Essai sur la peinture, Salons de 1759, 1761, 1763, édition de G. May, J. et A.-M. Chouillet.

Paris : Hermann.

GRIMM, DIDEROT, RAYNAL et alii (1880). Correspondance Littéraire, édition de Maurice Tourneux. Paris : Garnier frères, tome XIII.

\section{Textes critiques}

ARON, Paul, SAINT-JACQUES, Denis, VIALA, Alain (2010). Dictionnaire du littéraire, Paris : PUF, «Quadrige». 
BOUILLON, Jean-Paul : « Mise au point théorique et méthodologique », Revue d'Histoire Littéraire de la France, 1980, nº 6, « Littérature et Peinture ».

DEAN, Philippe. « Deux sortes de peintures », Recherches sur Diderot et sur l'Encyclopédie, numéro 22, 1997.

DIECKMANN, Herbert (1951). Inventaire du fonds Vandeul, Droz.

DUPRAT, Annie. « David, de la polémique à la gloire. Autour du Saint Roch du Bureau de la Santé de Marseille (1780) », Sociétés \& Représentations ํㅡㄹ, 2008/2, Paris : Publications de la Sorbonne, pp. 171-182.

MAY, Gita : "Diderot et Roger de Piles ", Publications of Modern Language Association, vol. 85, No. 3, May, 1970, pp. 444-455.

MODICA, Massimo. « Diderot philosophe et critique d'art », Recherches sur Diderot et sur l'Encyclopédie, 33, 2002.

REBEJKOW, Jean-Christophe : « Diderot, les Salons de 1767, de 1769 et la question du luxe », Diderot Studies XXIX (2003), pp. 65-82.

REBEJKow, Jean-Christophe : «La poésie dans les Salons de Diderot », Studi settecenteschi, vol. 25-26, 2005, pp. 203-230.

TLF [Trésor de la Langue française informatisé] [consulté le 11 juin 2014] <http://atilf.atilf.fr/ dendien/scripts/tlfiv5/affart.exe?19;s=2757459330;?b=0>

\section{NOTES}

1. En ce qui concerne Diderot, nous avons eu recours aux éditions suivantes: Diderot: Euvres complètes, édition publiée par Jules Assézat et Maurice Tourneux, Paris: Garnier frères, 1875-1877, 20 vol. (ci-après : AT, suivi du numéro du tome, en caractères romains, et de la page, en caractères arabes); Diderot: CEuvres complètes, édition publiée sous la direction de Roger Lewinter, Paris : Le Club français du livre, 1969-1973, 15 vol. (ci-après : LEW, suivi du numéro du tome, en caractères romains, et de la page, en caractères arabes). L'Essai sur la peinture, les Salons de 1759, 1761, 1763 (Paris: Hermann, 2003), ont fait l'objet d'une édition critique, richement annotée, par Gita May et Jacques Chouillet (ci-après, Hermann, suivi du numéro de la page); le texte des salons, en particulier, est mieux établi dans cette dernière édition.

2. Cf. Le Dictionnaire du littéraire, sous la direction de P. Aron, D. Saint Jacques, A. Viala, Paris : PUF, "Quadrige ", 2010, p. 116.

3. Correspondance Littéraire, édition de Maurice Tourneux, Paris : Garnier frères, tome XIII, 1880, p. 468 (ci-après : CL, suivi du numéro du tome, en caractères romains, et de la page, en caractères arabes ; ici : CL, XIII, p. 468).

4. Dans l'édition du Journal de Barbier le terme "chronique » semble être introduit par l'éditeur du XIX ${ }^{\mathrm{e}}$ s. (cf. La Chronique de la Régence et du règne de Louis XV (1718-1705) ou Journal de Barbier, Paris : Charpentier, 1857 ; cette «Chronique », dit l'éditeur, est « rédigée au jour le jour » ( Introduction, $\mathrm{p}$. VIII)).

5. Cf. Le dictionnaire du littéraire, sous la direction de P. Aron, D. Saint Jacques, A. Viala : « Dans le vocabulaire journalistique, la Chronique propose des articles d'actualité paraissant régulièrement, sur un sujet donné, et le mot s'applique notamment à la critique » (Paris : PUF, "Quadrige», 2010, p. 116). Le TLF donne aussi cette acception: «3. Article de journal ou de revue, émission de radio ou de télévision, produits régulièrement et consacrés à des informations, des commentaires sur un sujet précis. Une Chronique littéraire, politique, sportive ». 
6. Cf. Jean-Paul Bouillon : «L'étude des genres amènerait elle aussi à dépasser le culte exclusif des "auteurs" pour voir, dans la perspective d'une analyse sociologique de ces "formes", comment l'article de presse ou la notice de dictionnaire, la Chronique d'art (Burty, Geffroy), le compte rendu d'exposition (les Salons), le guide de musée (Gautier), le récit de voyage, la monographie (Champfleury, Goncourt), l'étude historique (Thoré, Chesneau), le texte polémique (Silvestre, Mirbeau), le manifeste (Duranty, mais aussi Courbet ou Manet), le recueil d'aphorismes (Dolent), le roman sur l'art (Burty, Goncourt, Zola), le roman d'art, ou même le genre tout à fait particulier de la correspondance d'art (Pissarro, Van Gogh, Cézanne), constituent autant de "catégories" qui répondent le plus souvent à des lois précises, et à l'intérieur desquelles les rapports de filiation sont à étudier " ("Mise au point théorique et méthodologique", Revue d'Histoire Littéraire de la France, 1980, nº 6, "Littérature et Peinture", p. 897).

7. Gita May, « Diderot et Roger de Piles ", Publications of Modern Language Association, vol. 85, No. 3 (Mai, 1970), pp. 444-455; ici p. 444.

8. L'association est sous-entendue par Philippe Dean («Deux sortes de peintures », Recherches sur Diderot et sur l'Encyclopédie, numéro 22, 1997, p. 44). Massimo Modica pense que Grimm avait précédé Diderot dans cette activité (les salons) par ses Chroniques des salons de 1753, 1755, 1757 («Diderot philosophe et critique d'art», Recherches sur Diderot et sur l'Encyclopédie, 33 (2002), p. 77). Voir aussi Annie Duprat qui parle de «Chronique artistique» à propos des salons de Diderot («David, de la polémique à la gloire. Autour du Saint Roch du Bureau de la Santé de Marseille (1780)» Sociétés \& Représentations 2008/2 ( $\left.n^{\circ} 26\right)$, Paris : Publications de la Sorbonne, pp. 171-182).

9. J. Chouillet note justement que Diderot en viendra «à indiquer lui-même des références en marge (voir le Salon de 1761), mais il n'ira jamais jusqu'à une mise en forme répertoriée et numérotée » (op.cit., p. 89).

10. Nous ne disposons pour ce passage du Salon de 1761 que du texte remanié par Grimm (cf. J. Chouillet, op. cit., p. 111, pp. 164-65 ; AT, X, p. 151).

11. Pour l'éventuel lecteur intéressé par cet aspect, nous nous permettons de renvoyer à notre article, «Le Salon de 1767 ou les métamorphoses de l'esthétique de Diderot », Poetica, 39 Band, Heft 1-2 (2007), pp. 89-109.

12. $C f$. sur ce point, notre article, "La poésie dans les Salons de Diderot ", Studi settecenteschi, vol. 25-26 (2005), pp. 203-230.

13. Salon de 1761 (AT, X, p. 127 ; LEW, V, p. 73).

14. La Satire contre le luxe, que Diderot songea un temps à intégrer dans le Salon de 1767, comme en font foi l'édition Naigeon et la copie de ce Salon conservée au château de Coppet, ne figure pas dans le manuscrit autographe, tel qu'il est parvenu jusqu'à nous. Notons que les thèmes du luxe, des artistes corrompus par l'or, traversent le Salon de 1767, la Satire contre le luxe à la manière de Perse, les Regrets sur ma vieille robe de chambre, le Salon de 1769 (cette corruption explique selon lui la pauvreté de ce dernier salon). Voir notre article: «Diderot, les Salons de 1767, de 1769 et la question du luxe ", Diderot Studies XXIX (2003), 65-82.

15. Cf. l'Introduction de Roger Lewinter aux Regrets sur ma vieille robe de chambre (LEW, VIII, p. 9).

16. Le tableau représentant Septime Sévère, Salon de 1769, AT, XI, pp. 439-440.

17. «Le Greuze vient de faire un tour de force. Il s'est tout à coup élancé de la bambochade dans la grande peinture; et avec succès, autant que je m'y connais » (A Falconet, Juillet 1767, AT, XVIII, p. 249).

18. Cf. Herbert Dieckmann, Inventaire du fonds Vandeul, Droz, 1951, p. 91 (Bibliothèque Nationale de France, Paris, ms. n.a.fr. 13.767), fol. 53-78 ; ce manuscrit est publié in LEW, VII, avec une belle introduction de Roger Lewinter).

19. Cf. également, sur cette affaire, A. M. Wilson, Diderot, Paris : Laffont, "Bouquins ", 1984, pp. $480-81$. 
20. Cette juxtaposition de deux points de vue différents qui prédomine dans les salons suivants était annoncée, dans le Salon de 1769, par cette remarque de Diderot, qui fait part de la diversité (voire de l'incompatibilité) de certains de ses jugements : «Il faut être vrai, mon ami, et vous donner en même temps un excellent exemple de la manière dont on est diversement affecté d'un même morceau en différents instants ». annonce les avis contradictoires des salons suivants. » (AT, XI, 435).

21. On comparera avec intérêt les variantes entre le manuscrit autographe du Salon de 1761, et sa révision par Grimm dans la Correspondance Littéraire (cf. l'édition de ce salon par J. Chouillet, Hermann, 2003, op. cit.).

22. Ces notes sont « clairement indiquées par des tirets » selon Roger Lewinter (Introduction, LEW, XIII, 82). Dans son édition, la typographie, qui met ces notes en italiques, rend plus aisée que dans AT la lecture de ce salon (LEW, XIII, pp. 85-127, publié d'après deux copies de la Correspondance Littéraire).

\section{RÉSUMÉS}

$\mathrm{Au} \mathrm{XVIII}^{\mathrm{e}}$ siècle, la Chronique est considérée comme un simple récit d'évènements passés classés dans un ordre chronologique. Dans l'article *Chronique de l'Encyclopédie, Diderot la dévalorise, et semble opposer le chroniqueur à l'historien qui s'interroge avec profit sur les liens entre les évènements. Ce n'est qu'au XIX ${ }^{\mathrm{e}}$ siècle que le terme prend son sens actuel. Cependant, par son attention aux débats de l'époque sur l'art dont il fait écho dans les salons, par son activité de journaliste pour la Correspondance Littéraire de Grimm, Diderot s'apparente à un chroniqueur. Dans cette perspective, il nous a paru intéressant de nous pencher sur les derniers salons de Diderot, en particulier sur le Salon de 1769, qui juxtapose sans lien deux chroniques de nature différente, la Chronique artistique et la "Chronique du quotidien », cette dernière ne nourrissant pas la réflexion esthétique de Diderot. Ce procédé est repris, avec des variantes (il s'agit de deux chroniques d'art qui ne dialoguent pas entre elles), dans les salons suivants : ceux de 1771, 1775, 1781. Dans les derniers salons, cette juxtaposition de deux chroniques signale de façon exemplaire l'abandon du projet esthétique de Diderot : rivaliser avec le peintre.

In the 18th Century, the Chronicle is considered as a simple narrative of past events classified in a chronological order. In the article *Chronique of the Encyclopédie, Diderot depreciates it. Only in the 19th Century, the term got its current meaning. However, by its attention on the debates of his time which he echoes in his Salons published in Grimm's Correspondance Littéraire, Diderot may be considered as an author of chronicles. In this perspective, it seemed interesting to us to pay attention to the last Salons, particularly to the Salon de 1769, which juxtaposes without links between them two chronicles of different nature, art chronicle, and a chronicle of the life, without links to esthetical problems. The last Salons, indicates in a exemplary way the abandonment of the esthetic project of Diderot: to compete with the painter.

\section{INDEX}

Mots-clés : Chronique, Diderot (Denis), peinture, Salon, description

Keywords : Chronicle, Diderot (Denis), painting, Salon, description 


\section{AUTEUR}

\section{JEAN-CHRISTOPHE REBEJKOW}

Champigny (France)

Jean-Christophe.Rebejkow[at]ac-creteil.fr 